Article

\title{
Alicyclic $\beta$ - and $\gamma$-Amino Acids: Useful Scaffolds for the Stereocontrolled Access to Amino Acid-Based Carbocyclic Nucleoside Analogs
}

\author{
Attila Márió Remete ${ }^{1,2}$ and Loránd Kiss ${ }^{1,2, *}$ \\ 1 Institute of Pharmaceutical Chemistry, University of Szeged, Eötvös u. 6, H-6720 Szeged, Hungary; \\ remete.attila@pharm.u-szeged.hu \\ 2 Interdisciplinary Excellence Centre, Institute of Pharmaceutical Chemistry, University of Szeged, Eötvös u. 6, \\ H-6720 Szeged, Hungary \\ * Correspondence: kiss.lorand@pharm.u-szeged.hu; Tel.: +36-308904092
}

Received: 10 December 2018; Accepted: 23 December 2018; Published: 3 January 2019

\begin{abstract}
Stereocontrolled synthesis of some amino acid-based carbocyclic nucleoside analogs containing ring $C=C$ bond has been performed on $\beta$ - and $\gamma$-lactam basis. Key steps were $N$-arylation of readily available $\beta$ - or $\gamma$-lactam-derived amino ester isomers and amino alcohols with 5-amino-4,6-dichloropyrimidine; ring closure of the formed adduct with $\mathrm{HC}(\mathrm{OMe})_{3}$ and nucleophilic displacement of chlorine with various $\mathrm{N}$-nucleophiles in the resulting 6-chloropurine moiety.
\end{abstract}

Keywords: amino acids; nucleoside analogs; carbocycles; lactams

\section{Introduction and Aims}

In carbocyclic nucleoside analogs, a methylene group replaces the oxygen atom in the carbohydrate ring, thereby increasing stability towards hydrolases and phosphorylases. The synthesis of these molecules is an area of considerable interest to medicinal chemistry, thanks to their bioactivity. Within natural products, neplanocin A is an antitumor antibiotic, while aristeromycin has antibacterial and antiviral activities. With respect to synthetic compounds, (-)-carbovir (1) and abacavir (2) show anti-HIV activity (Scheme 1) while entecavir inhibits the hepatitis B virus [1-6]. Carbocyclic nucleoside analogs with a 6-membered ring received less attention. In their case, antiviral activity usually requires the presence of a $C=C$ bond in the ring $[1-3,5,7,8]$ (see 3 and 4), enabling the base to occupy a pseudoaxial position [1,5], but some (2-aminocyclohexyl)methanol derivatives (for example, 5 [9]) also exhibit bioactivity (Scheme 1).
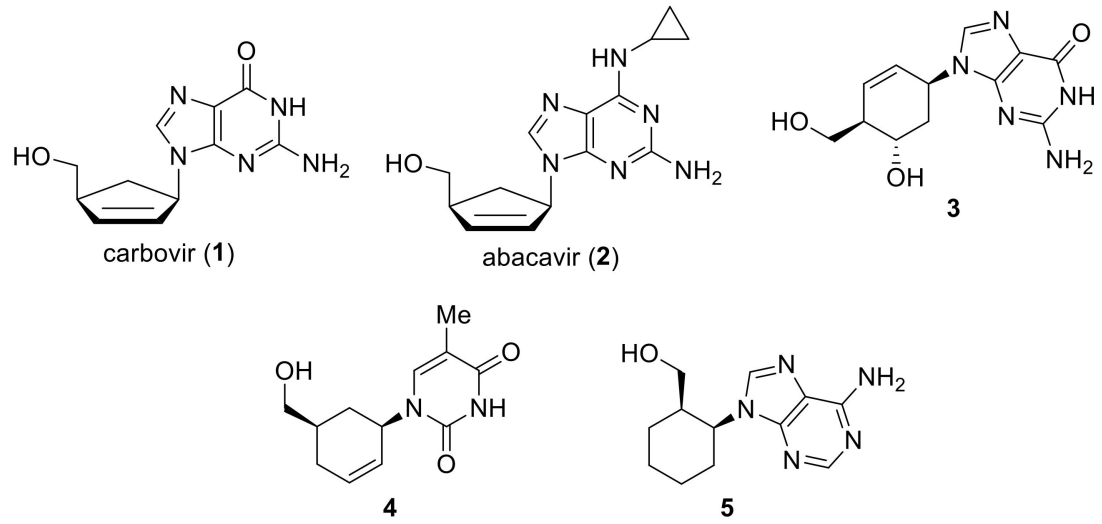

Scheme 1. Some bioactive nucleoside analogs. 
Cyclic $\beta$-amino acids have gained significant attention in the last few decades [10-14]. They can be found in natural products, such as peptidyl nucleoside antibiotics amipurimycin (6), chryscandin (7), blasticidin S (8) or gougerotin (9), and related analogous derivatives (Scheme 2) [15-17]. In the latter three nucleoside analogs, the sugar ring was replaced with a cyclic $\beta$-amino acid unit. Cyclic $\beta$-amino acids are also promising building blocks of new bioactive peptides [14-21] and many simple representatives show relevant biological activity (Scheme 3), such as the analgesic drug tilidine (10) or antifungal antibiotics cispentacin and icofungipen (11) [10-13].

Highly-functionalized cyclic $\gamma$-amino acid derivatives possessing multiple stereogenic centers are also of considerable importance in drug research. Neuraminidase inhibitors Peramivir (12, Scheme 3), Zanamivir and Oseltamivir and their modified analogs are used in the treatment of influenza [22], while Gabapentin [23] and CPP-115 [24] (13, Scheme 3) are anticonvulsant drugs.<smiles></smiles>
amipurimycin (6)<smiles>COc1ccc(C[C@H](N)C(=O)N[C@H]2O[C@H](C(=O)O)[C@@H](O)[C@H](C)[C@H]2O)cc1</smiles>

chryscandin (7)<smiles>CNCC(=O)NC(O)C(=O)N[C@H]1C(O)[C@H](O)C(n2ccc(N)nc2=O)O[C@H]1C(N)=O</smiles>

Scheme 2. Some bioactive $\beta$-amino acid-based nucleoside analogs.<smiles>CCOC(OCC)(c1ccccc1)C1CCC=CC1N(C)C</smiles>

tilidine (10)<smiles>C=C1CC(N)C(C(=O)O)C1</smiles>

icofungipen (11)<smiles>CCC(CC)[C@H](NC(=N)N)C1C(O)[C@H](C(=O)O)C[C@H]1NC(N)=O</smiles>

peramivir (12)<smiles>N[C@H]1C[C@@H](C(=O)O)C/C1=C(/F)C(=O)O</smiles>

CPP-115 (13)

Scheme 3. Examples of bioactive cyclic amino acid derivatives.

\section{Results and Discussion}

Taking into account the importance of carbocyclic nucleoside analogs and the bioactivity of peptidyl nucleoside antibiotics containing $\beta$-amino acids, our aim was the synthesis of new carbocyclic nucleoside analogs with an amino acid moiety on a $\beta$ - and $\gamma$-lactam basis. This pathway is similar to the first synthesis of carbovir from unsaturated $\gamma$-lactam $( \pm$ )-14 (also known as Vince lactam) [25-27]. The synthesis of some 6-membered carbocyclic nucleoside analogs containing $\gamma$-amino alcohol was also planned. 
Our synthetic work started with the opening of the heteroring of racemic Vince lactam ( \pm )-14 [28]. Construction of the nucleobase part on the resulting amino ester ( \pm )-15 was accomplished in three steps. First, compound ( \pm )-15 was subjected to $N$-arylation with 5-amino-4,6-dichloropyrimidine to furnish ( \pm )-16. This process was accompanied by $\mathrm{C}=\mathrm{C}$ bond migration thanks to the basic conditions, enabling the formation of a more stable conjugated $\pi$-system. Then, reaction with trimethyl orthoformate generated the second heteroring. The remaining chlorine atom of the obtained nucleoside analog ( \pm )-17 was then replaced with $N$-nucleophiles to obtain adenosine analogs $( \pm)-\mathbf{1 8},( \pm)-\mathbf{1 9}$ and $( \pm)-20$ (Scheme 4 ). It is worth to note that compound $( \pm)-19$ contains a cyclopropylamino group similar to abacavir, while the azido group of compound ( \pm )-20 enables many further transformations (e.g., triazole formation).

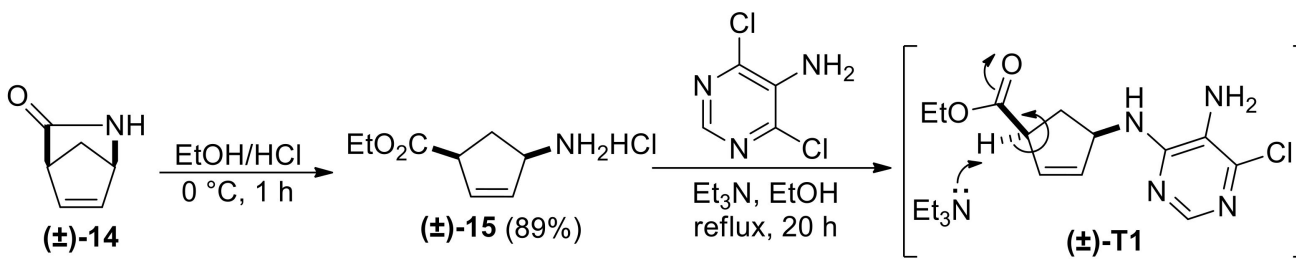<smiles>CCOC(=O)C1=CCC(Nc2ncnc(Cl)c2N)C1</smiles>

Scheme 4. Synthesis of cyclic $\gamma$-amino acid-based nucleoside analogs.

We continued our synthetic work with ethyl cis $\beta$-amino ester hydrochloride $( \pm)$-22 obtained from $\beta$-lactam ( \pm )-21 [29,30]. Lactam ring opening, construction of the nucleobase moiety, and aromatic nucleophilic substitution resulted in nucleoside analogs ( \pm -25 and ( \pm )-26. From ethyl trans $\beta$-amino ester hydrochloride $( \pm)-\mathbf{2 8}[31,32]$, azidonucleoside $( \pm)-\mathbf{3 1}$ was prepared in a similar way (Schemes 5 and 6). 


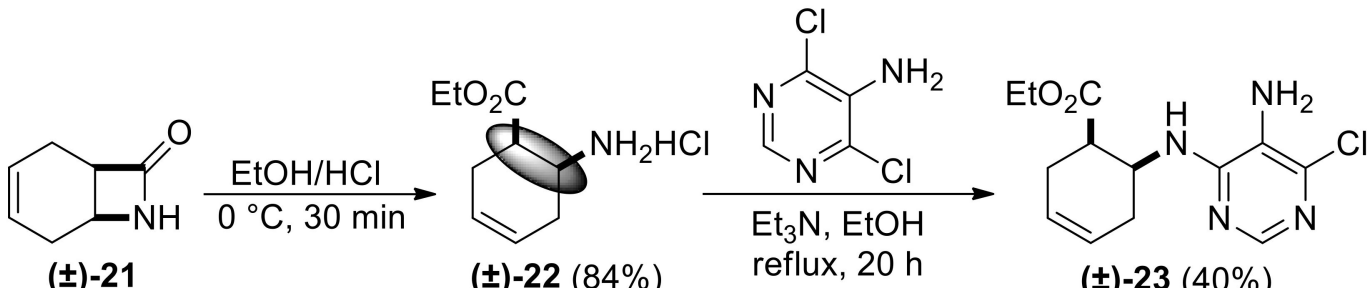

$( \pm)-21$

(士)-22 (84\%) reflux, $20 \mathrm{~h}$

(士)-23 (40\%)

$\mathrm{HC}(\mathrm{OMe})_{3}$, cat. $\mathrm{MsOH}, 20^{\circ} \mathrm{C}, 6 \mathrm{~h}$<smiles>CCOCCN(CC)C1CC=CCC1</smiles>

(士)-25 (51\%)
4 eq. $\mathrm{NaN}_{3}$, cat. $\mathrm{AcOH}$, cat. $\mathrm{Et}_{3} \mathrm{~N}$ $\mathrm{THF} / \mathrm{H}_{2} \mathrm{O}$, reflux, $20 \mathrm{~h}$

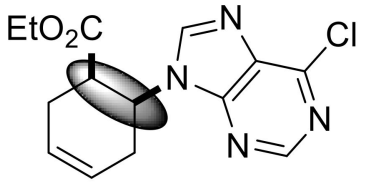

(士)-24 (76\%)

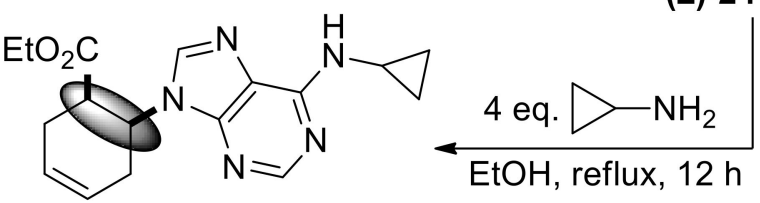

(士)-26 (62\%)

Scheme 5. Synthesis of $\beta$-amino acid-based nucleoside analogs from ethyl cis-2-aminocyclohex4-enecarboxylate hydrochloride $( \pm)$-22.

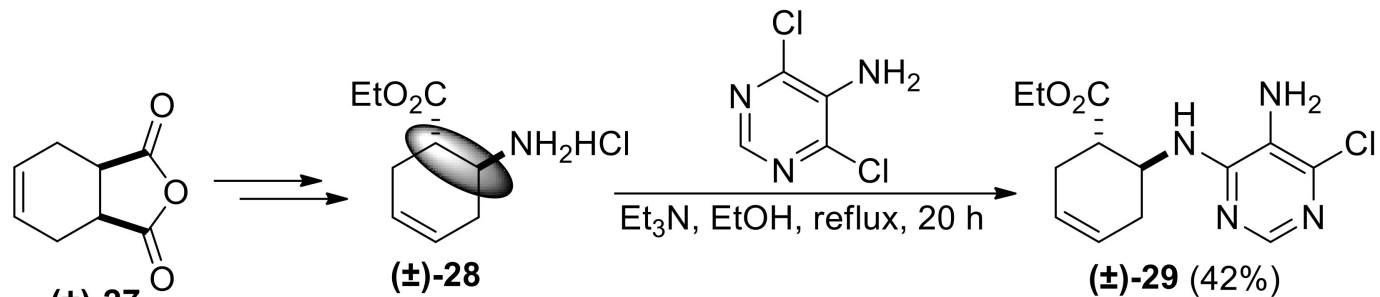

(士)-27

(士)-28

$\mathrm{HC}(\mathrm{OMe})_{3}$, cat. $\mathrm{MsOH}, 20^{\circ} \mathrm{C}, 6 \mathrm{~h} \downarrow$

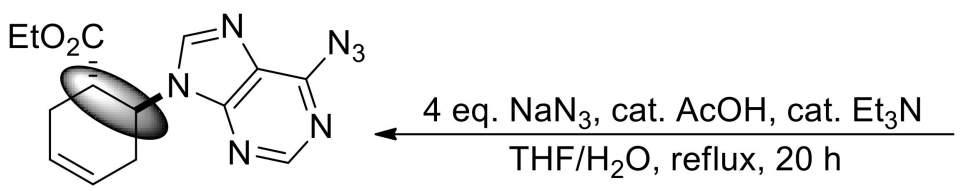

(士)-31 (68\%)<smiles>CCOC(=O)[C@H]1CC=CCC1n1cnc2c(Cl)ncnc21</smiles>

(士)-30 (58\%)

Scheme 6. Synthesis of $\beta$-amino acid-based nucleoside analogs from ethyl trans-2-aminocyclohex4-enecarboxylate hydrochloride $( \pm)$-28.

Note that the synthetic protocol took place with stereocontrol in both cases. Since the configuration of the chiral centers are not affected during the syntheses, their integrity is conserved and therefore, the cis-amino acid starting material led to the corresponding carbanucleoside analog in which the relative configuration of the groups is cis, while the trans-amino acid provided the carbocyclic nucleobase analog with trans relative steric arrangement of the ester and the heterocycle.

Analogous treatment of ethyl cis-2-aminocyclohex-3-enecarboxylate hydrochloride $( \pm)$-33 (a regioisomer of $( \pm$ )-22), obtained from $\beta$-lactam $( \pm)$-32 [33,34], resulted in nucleoside analog $( \pm$ )-35, the $\mathrm{C}=\mathrm{C}$ regioisomer of compound $( \pm)-24$ (Scheme 7). 


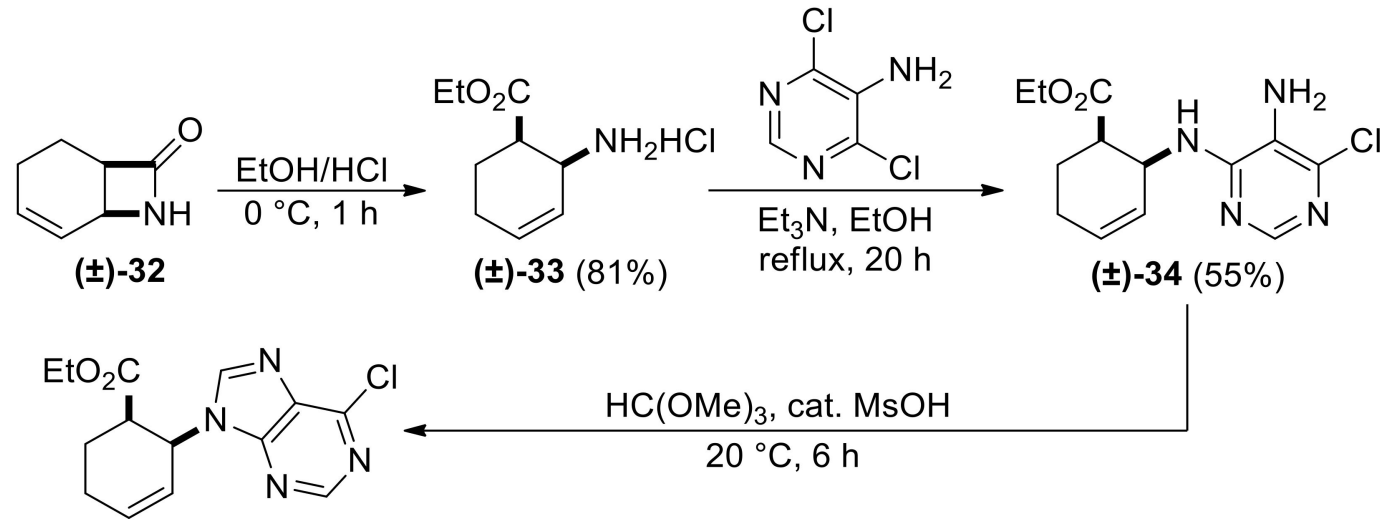

$( \pm)-35(68 \%)$

Scheme 7. Synthesis of $\beta$-amino acid-based nucleoside analog $( \pm)$-35 from ethyl cis-2-aminocyclohex3-enecarboxylate hydrochloride $( \pm)$-33.

In order to synthesize compounds with a five-membered carbocycle, the strategy was also extended to $\beta$-lactam $( \pm)$-36. Nucleoside analog ( \pm )-39 was obtained successfully using the protocol described above for the six-membered analogs, although both nucleobase construction steps had lower yields (Scheme 8).

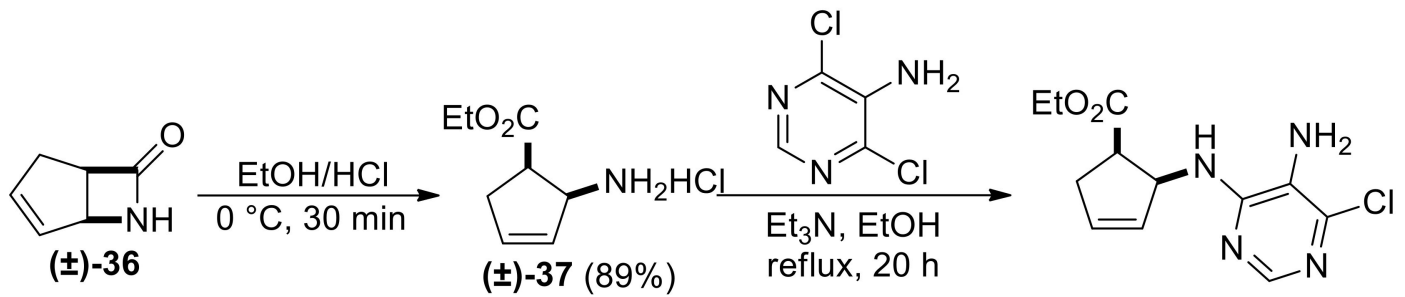

(士)-38 (24\%)<smiles>CCOCC1CC=CC1n1cnc2c(Cl)ncnc21</smiles>

( \pm )-39 (39\%)

Scheme 8. Synthesis of $\beta$-amino acid-based nucleoside analog ( \pm )-39 with a 5-membered carbocycle.

Taking into account the bioactivity of compounds $3,( \pm)-4$ and $( \pm)-5$, the synthesis of similar molecules was attempted. Reduction of $\beta$-amino acids $( \pm)-40$ and $( \pm)-44$ with $\mathrm{LiAlH}_{4}$ [32] afforded $\gamma$-amino alcohols $( \pm)$-41 and $( \pm)$-45, which were further reacted with 5-amino-4,6-dichloropyrimidine. Ring closing with trimethyl orthoformate in the last step yielded, through stereocontrol, nucleoside analogs ( \pm )-43 and $( \pm$ )-47 (Scheme 9). These compounds show high structural similarity to bioactive compound $( \pm)-5$. 


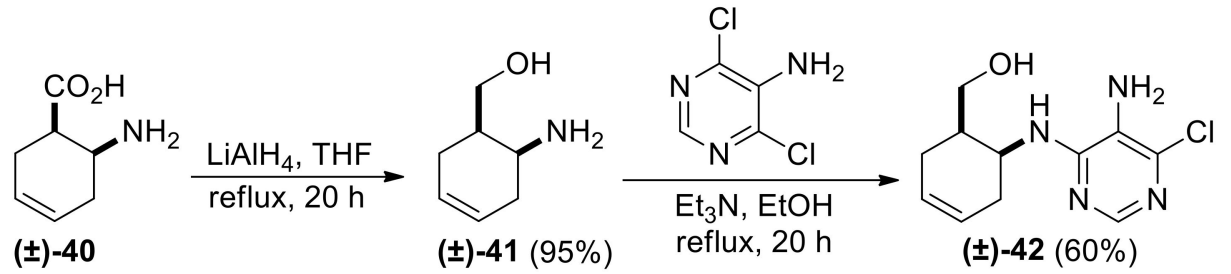<smiles>NC1CC=CCC1C(=O)O</smiles>

$( \pm)-44$

$\mathrm{LiAlH}_{4}, \mathrm{THF}$ reflux, $20 \mathrm{~h}$

$$
\checkmark
$$<smiles>NC1CC=CCC1CO</smiles>

(士)-45 (68\%)<smiles>CCN(CC)CC</smiles>
reflux, $20 \mathrm{~h}$

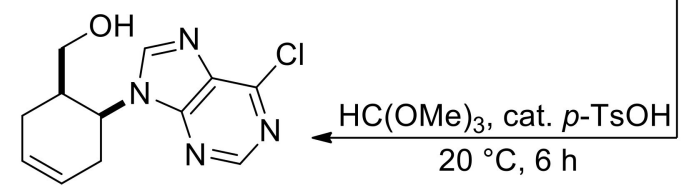

(士)-43 (76\%)

Scheme 9. Synthesis of unsaturated carbanucleoside isomers $( \pm)-43$ and $( \pm)-47$ analogs of carbocyclic nucleoside $( \pm)-5$.

\section{Conclusions and Outlook}

A stereocontrolled synthetic pathway was developed to prepare new carbocyclic nucleoside analogs containing a ring olefin bond with a $\beta$-amino acid, $\gamma$-amino acid or $\gamma$-amino alcohol moiety from readily available $\beta$ - and $\gamma$-lactams (across the amino acid isomers). The structure of the starting cycloalkene amino acids determined the configuration of the stereogenic centers of the products. 6- Nucleoside analogs containing the chloropurine moiety proved to be useful intermediates in various reactions with nucleophiles to access substituted nucleobases. Taking into consideration our widespread experiences in selective and controlled functionalization of versatile unsaturated cyclic amino acid derivatives [35-38], further studies in order to investigate the possible functionalization of the ring olefin bond of product nucleoside analogs are currently being investigated in our laboratory. Furthermore, based on our experiences in enzymatic resolution of various bicyclic $\beta$ - and $\gamma$-lactams $[39,40]$, as well as on enzymatic ester hydrolysis methodologies [41], synthesis of enantiomerically pure substances will be performed.

\section{Materials and Methods}

\subsection{General Information}

Chemicals were purchased from Sigma-Aldrich (Budapest, Hungary). Solvents were used as received from the suppliers. Amino ester hydrochlorides ( \pm )-15 [28], ( \pm )-22 [29,30], ( \pm$)-28$ [31,32], ( \pm )-33 [33,34], ( \pm -37 [42] and $\gamma$-amino alcohols ( \pm -41, ( \pm -45 [32] were synthesized according to literature. The ${ }^{1} \mathrm{H}-\mathrm{NMR}$ and ${ }^{13} \mathrm{C}-\mathrm{NMR}$ spectra of all new compounds are available in Supplementary Materials.

4.1.1. General Procedure for N-Arylation of Amino Ester Hydrochlorides with 5-Amino-2,6-Dichloropyrimidine

To a solution of the amino ester hydrochloride (10 mmoles) in EtOH (30 mL), 5-amino-2, 6-dichloropyrimidine (10 mmoles) and Et3N (30 mmoles) were added, then the mixture was treated at reflux temperature for $20 \mathrm{~h}$. After cooling to room temperature, the reaction mixture was concentrated 
under reduced pressure and the residue was taken up in EtOAc $(100 \mathrm{~mL})$. The organic layer was washed with water $(3 \times 50 \mathrm{~mL})$, dried with $\mathrm{Na}_{2} \mathrm{SO}_{4}$, and concentrated under reduced pressure. The crude product was purified by column chromatography on silica gel (eluent: n-hexane-EtOAc 2:1).

\subsubsection{General Procedure for $N$-Arylation of $\gamma$-Amino Alcohols with 5-Amino-2,6-Dichloropyrimidine}

To a solution of the $\gamma$-amino alcohol (8 mmoles) in EtOH (25 mL), 5-amino-2,6-dichloropyrimidine ( 8 mmoles) and $\mathrm{Et}_{3} \mathrm{~N}$ (24 mmoles) were added, then the mixture was kept at boiling temperature for $20 \mathrm{~h}$. After cooling to room temperature, the reaction mixture was concentrated under reduced pressure and the residue was taken up in EtOAc $(100 \mathrm{~mL})$. The organic layer was washed with water $(3 \times 40 \mathrm{~mL})$, dried with $\mathrm{Na}_{2} \mathrm{SO}_{4}$ and concentrated under reduced pressure. The crude product was purified by column chromatography on silica gel (eluent: $n$-hexane-EtOAc 1:2).

\subsubsection{General Procedure for the Formation of the Purine Skeleton of Amino Ester Nucleoside Analogs}

To a solution of amino ester ( 2 mmoles) in trimethyl orthoformate $(5 \mathrm{~mL})$, a catalytic amount of methanesulfonic acid or $p$ - $\mathrm{TsOH}(30 \mathrm{mg})$ was added. After stirring at $20{ }^{\circ} \mathrm{C}$ for $6 \mathrm{~h}$, the reaction mixture was diluted with EtOAc $(25 \mathrm{~mL})$ and washed with saturated aqueous $\mathrm{NaCl}$ solution $(3 \times 15 \mathrm{~mL})$. The organic phase was dried with $\mathrm{Na}_{2} \mathrm{SO}_{4}$ and concentrated under reduced pressure. The crude product was purified by column chromatography on silica gel (eluent: $n$-hexane-EtOAc 1:1).

4.1.4. General Procedure for the Formation of the Purine Skeleton of Amino Alcohol Nucleoside Analogs

To a solution of amino alcohol nucleoside analog $(1 \mathrm{mmol})$ in trimethyl orthoformate $(4 \mathrm{~mL})$, a catalytic amount of $p-\mathrm{TsOH}(20 \mathrm{mg})$ was added. After stirring at $20{ }^{\circ} \mathrm{C}$ for $6 \mathrm{~h}$, the reaction mixture was diluted with EtOAc $(20 \mathrm{~mL})$ and washed with saturated aqueous $\mathrm{NaCl}$ solution $(3 \times 15 \mathrm{~mL})$. The organic phase was dried with $\mathrm{Na}_{2} \mathrm{SO}_{4}$ and concentrated under reduced pressure. The crude product was purified by column chromatography on silica gel (eluent: $n$-hexane-EtOAc 1:2).

\subsubsection{General Procedure for the Introduction of the Azido Group}

To a solution of 6-chloropurinyl nucleoside analog $(150 \mathrm{mg})$ in $\mathrm{THF} / \mathrm{H}_{2} \mathrm{O}(10 \mathrm{~mL}, 4: 1)$, sodium azide ( 4 eq.), acetic acid ( 3 drops), and $\mathrm{Et}_{3} \mathrm{~N}$ ( 4 drops) were added. After heating at reflux temperature for $20 \mathrm{~h}$, the reaction mixture was diluted with EtOAc $(20 \mathrm{~mL})$ and washed with water $(2 \times 15 \mathrm{~mL})$. The organic phase was dried with $\mathrm{Na}_{2} \mathrm{SO}_{4}$ and concentrated under reduced pressure. The crude product was purified by column chromatography on silica gel (eluent: $n$-hexane-EtOAc 1:2).

\subsubsection{General Procedure for the Introduction of the Cyclopropylamino Group}

To a solution of 6-chloropurinyl nucleoside analog $(150 \mathrm{mg})$ in $\mathrm{EtOH}(10 \mathrm{~mL})$, cyclopropylamine (4 eq.) was added. After the mixture was kept at boiling temperature for $12 \mathrm{~h}$, the reaction mixture was concentrated under reduced pressure. The crude product was purified by column chromatography on silica gel (eluent: $n$-hexane-EtOAc 1:1).

\subsection{Synthesis of Methylamino Compound $( \pm) \mathbf{- 1 8}$}

To a solution of 6-chloropurinyl nucleoside analogue $( \pm)-\mathbf{1 7}(150 \mathrm{mg})$ in EtOH $(10 \mathrm{~mL}), \mathrm{MeNH}_{2}$ (4 eq.) was added. After heating under reflux for $20 \mathrm{~h}$, the reaction mixture was concentrated under reduced pressure. The crude product was purified by column chromatography on silica gel (eluent: n-hexane-EtOAc 1:1). 
<smiles>CCOC(=O)C1=CCC(Nc2ncnc(Cl)c2N)C1</smiles>

Ethyl $\left(S^{*}\right)$-4-((5-amino-6-chloropyrimidin-4-yl)amino)cyclopent-1-ene-1-carboxylate, $( \pm)$-16.

Brownish white solid, m.p. $121-123{ }^{\circ} \mathrm{C}, 40 \% ;{ }^{1} \mathrm{H}-\mathrm{NMR}\left(\mathrm{CDCl}_{3}, 400 \mathrm{MHz}\right): \delta(\mathrm{ppm})=1.30\left(\mathrm{t}, 3 \mathrm{H}, \mathrm{CH}_{3}\right.$, $J=7.14 \mathrm{~Hz}), 2.43-2.59\left(\mathrm{~m}, 2 \mathrm{H}, \mathrm{CH}_{2}\right), 3.00-3.16\left(\mathrm{~m}, 2 \mathrm{H}, \mathrm{CH}_{2}\right), 3.44\left(\mathrm{brs}, 2 \mathrm{H}, \mathrm{NH}_{2}\right), 4.17-4.25(\mathrm{~m}, 2 \mathrm{H}$, $\left.\mathrm{OCH}_{2}\right), 4.79-4.86(\mathrm{~m}, 1 \mathrm{H}, \mathrm{H}-4), 5.08(\mathrm{~d}, 1 \mathrm{H}, \mathrm{N}-\mathrm{H}, J=5.76 \mathrm{~Hz}), 6.76-6.78(\mathrm{~m}, 1 \mathrm{H}, \mathrm{H}-2), 8.08(\mathrm{~s}, 1 \mathrm{H}, \mathrm{Ar}-\mathrm{H})$; ${ }^{13} \mathrm{C}-\mathrm{NMR}$ (DMSO, $\left.100 \mathrm{MHz}\right): \delta(\mathrm{ppm})=15.0,39.3,41.2,51.3,60.7,124.6,135.1,137.7,142.6,146.4,152.0$, 164.9; MS (ES, pos) $m / z=283(\mathrm{M}+1)$.<smiles>CCOC(=O)C1=CCC(n2cnc3c(Cl)ncnc32)C1</smiles>

Ethyl ( $\left.S^{*}\right)-4-(6-c h l o r o-9 H-p u r i n-9-y l) c y c l o p e n t-1-e n e-1-c a r b o x y l a t e, ~( \pm)-17$.

Yellowish white solid, m.p. $83-85{ }^{\circ} \mathrm{C}, 81 \%$; ${ }^{1} \mathrm{H}-\mathrm{NMR}$ (DMSO, $\left.400 \mathrm{MHz}\right): \delta=1.20\left(\mathrm{t}, 3 \mathrm{H}, \mathrm{CH}_{3}\right.$, $J=7.08 \mathrm{~Hz}), 2.93-3.05\left(\mathrm{~m}, 2 \mathrm{H}, \mathrm{CH}_{2}\right), 3.06-3.20\left(\mathrm{~m}, 2 \mathrm{H}, \mathrm{CH}_{2}\right), 4.09-4.17\left(\mathrm{~m}, 2 \mathrm{H}, \mathrm{OCH}_{2}\right), 5.38-5.47$ (m, 1H, H-4), 6.70-6.81 (m, 1H, H-2), 8.69 (s, 1H, Ar-H), 8.74 (s, 1H, Ar-H); ${ }^{13} \mathrm{C}-\mathrm{NMR}\left(\mathrm{CDCl}_{3}, 100 \mathrm{MHz}\right)$ : $\delta(\mathrm{ppm})=14.6,39.2,40.9,54.2,61.2,132.2,135.5,140.1,143.5,151.6,151.8,152.3,164.2$; MS (ES, pos) $m / z=293(\mathrm{M}+1)$.<smiles>CCOC(=O)C1=CCC(n2cnc3c(NC)ncnc32)C1</smiles>

Ethyl ( $\left.S^{*}\right)-4-(6-(m e t h y l a m i n o)-9 H-p u r i n-9-y l) c y c l o p e n t-1-e n e-1-c a r b o x y l a t e, ~( \pm)-18$.

Yellow oil, 68\%; ${ }^{1} \mathrm{H}-\mathrm{NMR}\left(\mathrm{CDCl}_{3}, 400 \mathrm{MHz}\right): \delta(\mathrm{ppm})=1.32\left(\mathrm{t}, 3 \mathrm{H}, \mathrm{CH}_{3}, J=7.12 \mathrm{~Hz}\right), 2.86-3.03(\mathrm{~m}, 2 \mathrm{H}$, $\left.\mathrm{CH}_{2}\right), 3.10-3.29\left(\mathrm{~m}, 5 \mathrm{H}, \mathrm{NCH}_{3}\right.$ and $\left.\mathrm{CH}_{2}\right), 4.20-4.31\left(\mathrm{~m}, 2 \mathrm{H}, \mathrm{OCH}_{2}\right), 5.41-5.50(\mathrm{~m}, 1 \mathrm{H}, \mathrm{H}-4), 6.16$ (brs, 1H, N-H), 6.86-6.89 (m, 1H, H-2), 7.75 (s, 1H, Ar-H), 8.41 (s, 1H, Ar-H); ${ }^{13} \mathrm{C}-\mathrm{NMR}\left(\mathrm{CDCl}_{3}, 100 \mathrm{MHz}\right)$ : $\delta(\mathrm{ppm})=14.6,39.3,41.1,50.6,58.9,61.1,120.4,135.5,136.1,137.7,140.5,153.4,155.9,164.5 ;$ MS (ES, pos) $m / z=288(\mathbf{M}+1)$.<smiles>CCOC(=O)C1=CCC(n2cnc3c(NC4CC4)ncnc32)C1</smiles>

Ethyl $\left(S^{*}\right)-4-(6-($ cyclopropylamino)-9H-purin-9-yl)cyclopent-1-ene-1-carboxylate, $( \pm)$-19.

Yellow oil, 53\%; ${ }^{1} \mathrm{H}-\mathrm{NMR}\left(\mathrm{CDCl}_{3}, 500 \mathrm{MHz}\right): \delta(\mathrm{ppm})=0.64-0.69\left(\mathrm{~m}, 2 \mathrm{H}, \mathrm{CH}_{2}\right), 0.90-0.97(\mathrm{~m}, 2 \mathrm{H}$, $\left.\mathrm{CH}_{2}\right), 1.32\left(\mathrm{t}, 3 \mathrm{H}, \mathrm{CH}_{3}, \mathrm{~J}=7.13 \mathrm{~Hz}\right), 2.86-3.08\left(\mathrm{~m}, 3 \mathrm{H}, \mathrm{CH}_{2}\right), 3.18-3.32\left(\mathrm{~m}, 2 \mathrm{H}, \mathrm{CH}_{2}, \mathrm{CH}\right), 4.21-4.28$ $\left(\mathrm{m}, 2 \mathrm{H}, \mathrm{OCH}_{2}\right), 5.35-5.43(\mathrm{~m}, 1 \mathrm{H}, \mathrm{H}-4), 6.03$ (brs, $\left.1 \mathrm{H}, \mathrm{N}-\mathrm{H}\right), 6.86-6.91(\mathrm{~m}, 1 \mathrm{H}, \mathrm{H}-2), 7.75(\mathrm{~s}, 1 \mathrm{H}, \mathrm{Ar}-\mathrm{H})$, 8.48 (s, 1H, Ar-H); ${ }^{13} \mathrm{C}-\mathrm{NMR}\left(\mathrm{CDCl}_{3}, 126 \mathrm{MHz}\right): \delta(\mathrm{ppm})=7.4,14.3,23.7,38.9,40.8,52.9,60.7,119.9$, 135.1, 137.6, 140.2, 153.2, 155.8, 164.1; MS (ES, pos) $m / z=314(\mathrm{M}+1)$. 
<smiles>CCOC(=O)C1=CCC(n2cnc3c(N)ncnc32)C1</smiles>

Ethyl ( $\left.S^{*}\right)-4-(6-a z i d o-9 H-p u r i n-9-y l) c y c l o p e n t-1-e n e-1-c a r b o x y l a t e, ~( \pm)-20$.

White solid, m.p. $145-147{ }^{\circ} \mathrm{C}, 68 \%$; ${ }^{1} \mathrm{H}-\mathrm{NMR}$ (DMSO, $\left.400 \mathrm{MHz}\right): \delta(\mathrm{ppm})=1.22\left(\mathrm{t}, 3 \mathrm{H}, \mathrm{CH}_{3}\right.$, $J=7.08 \mathrm{~Hz}), 2.97-3.09\left(\mathrm{~m}, 2 \mathrm{H}, \mathrm{CH}_{2}\right), 3.22-3.28\left(\mathrm{~m}, 2 \mathrm{H}, \mathrm{CH}_{2}\right), 4.12-4.23\left(\mathrm{~m}, 2 \mathrm{H}, \mathrm{OCH}_{2}\right), 5.53-5.64$ (m, 1H, H-4), 6.86-6.89 (m, 1H, H-2), 8.66 (s, 1H, Ar-H), 10.07 (s, 1H, Ar-H); ${ }^{13} \mathrm{C}-\mathrm{NMR}$ (DMSO, $100 \mathrm{MHz}): \delta(\mathrm{ppm})=15.0,34.9,39.2,55.1,61.0,121.2,134.5,136.3,141.8,142.8,143.8,146.3,164.5 ; \mathrm{MS}$ (ES, pos) $m / z=300(\mathbf{M}+1)$.<smiles>CCOC(=O)C1CC=CCC1Nc1ncnc(Cl)c1N</smiles>

Ethyl $\left(1 R^{*}, 6 S^{*}\right)-6$-((5-amino-6-chloropyrimidin-4-yl)amino)cyclohex-3-ene-1-carboxylate, $( \pm)$-23.

White solid, m.p. $120-121{ }^{\circ} \mathrm{C}, 42 \% ;{ }^{1} \mathrm{H}-\mathrm{NMR}\left(\mathrm{CDCl}_{3}, 400 \mathrm{MHz}\right): \delta(\mathrm{ppm})=1.28\left(\mathrm{t}, 3 \mathrm{H}, \mathrm{CH}_{3}, J=7.12 \mathrm{~Hz}\right)$, 2.26-2.38 (m, $\left.1 \mathrm{H}, \mathrm{CH}_{2}\right), 2.42-2.56\left(\mathrm{~m}, 2 \mathrm{H}, \mathrm{CH}_{2}\right), 2.62-2.73\left(\mathrm{~m}, 1 \mathrm{H}, \mathrm{CH}_{2}\right), 2.95-3.03(\mathrm{~m}, 1 \mathrm{H}, \mathrm{H}-1), 3.39$ (brs, 2H, NH$\left.)_{2}\right), 4.11-4.24\left(\mathrm{~m}, 2 \mathrm{H}, \mathrm{OCH}_{2}\right), 4.74-4.85$ (m, 1H, H-6), 5.66-5.87 (m, 3H, H-3, H-4, N-H), 8.08 (s, 1H, Ar-H); ${ }^{13 \mathrm{C}}$-NMR (DMSO, $\left.100 \mathrm{MHz}\right): \delta(\mathrm{ppm})=14.8,25.5,30.4,41.4,47.1,60.6,124.6,125.4$, $125.9,138.0,146.1,152.2,173.4$; MS (ES, pos) $m / z=297(\mathrm{M}+1), 299(\mathrm{M}+3)$.<smiles>CCOC(=O)C1CC=CCC1n1cnc2c(Cl)ncnc21</smiles>

Ethyl $\left(1 R^{*}, 6 S^{*}\right)-6$-(6-chloro-9H-purin-9-yl)cyclohex-3-ene-1-carboxylate, $( \pm)-24$.

Yellow oil, 76\%. ${ }^{1} \mathrm{H}-\mathrm{NMR}$ (DMSO, $\left.400 \mathrm{MHz}\right): \delta(\mathrm{ppm})=0.98\left(\mathrm{t}, 3 \mathrm{H}, \mathrm{CH}_{3}, J=7.12 \mathrm{~Hz}\right), 2.31-2.52(\mathrm{~m}$, $\left.2 \mathrm{H}, \mathrm{CH}_{2}\right), 2.74-2.84\left(\mathrm{~m}, 2 \mathrm{H}, \mathrm{CH}_{2}\right), 3.28-3.33(\mathrm{~m}, 1 \mathrm{H}, \mathrm{H}-1), 3.83-3.90\left(\mathrm{~m}, 2 \mathrm{H}, \mathrm{OCH}_{2}\right), 5.25-5.32(\mathrm{~m}, 1 \mathrm{H}$, H-6), 5.85-5.89 (m, 2H, H-3, H-4), 8.56 (s, 1H, Ar-H), 8.78 (s, 1H, Ar-H). ${ }^{13} \mathrm{C}-\mathrm{NMR}$ (DMSO, $100 \mathrm{MHz}$ ): $\delta(\mathrm{ppm})=14.5,25.4,29.3,42.0,51.2,61.2,125.3,126.4,131.2,146.6,149.9,152.2,153.0,172.4$; MS (ES, pos) $m / z=307(\mathbf{M}+1)$.<smiles>CCOC(=O)C1CC=CCC1n1cnc2c(N)ncnc21</smiles>

Ethyl $\left(1 R^{*}, 6 S^{*}\right)$-6-(6-azido-9H-purin-9-yl)cyclohex-3-ene-1-carboxylate, $( \pm)$-25.

White solid, m.p. ${ }^{130-132}{ }^{\circ} \mathrm{C}, 51 \%$; ${ }^{1} \mathrm{H}-\mathrm{NMR}$ (DMSO, $\left.400 \mathrm{MHz}\right): \delta(\mathrm{ppm})=1.03\left(\mathrm{t}, 3 \mathrm{H}, \mathrm{CH}_{3}\right.$, $J=7.10 \mathrm{~Hz}), 2.34-2.65\left(\mathrm{~m}, 2 \mathrm{H}, \mathrm{CH}_{2}\right), 2.78-3.00\left(\mathrm{~m}, 2 \mathrm{H}, \mathrm{CH}_{2}\right), 3.31-3.43(\mathrm{~m}, 1 \mathrm{H}, \mathrm{H}-1), 3.82-4.00$ $\left(\mathrm{m}, 2 \mathrm{H}, \mathrm{OCH}_{2}\right), 5.40-5.48(\mathrm{~m}, 1 \mathrm{H}, \mathrm{H}-6), 8.54(\mathrm{~s}, 1 \mathrm{H}, \mathrm{Ar}-\mathrm{H}), 10.13$ (s, 1H, Ar-H); ${ }^{13} \mathrm{C}-\mathrm{NMR}$ (DMSO, $100 \mathrm{MHz}): \delta(\mathrm{ppm})=14.6,25.4,29.7,42.3,51.5,61.3,125.3,126.4,129.4,130.4,135.3,142.8,147.9,170.5$; MS (ES, pos) $m / z=314(\mathrm{M}+1)$. 
<smiles>CCOC(=O)C1CC=CCC1n1cnc2c(NC3CC3)ncnc21</smiles>

Ethyl $\left(1 R^{*}, 6 S^{*}\right)$-6-(6-(cyclopropylamino)-9H-purin-9-yl)cyclohex-3-ene-1-carboxylate, $( \pm)$-26.

White solid, m.p. $128-129{ }^{\circ} \mathrm{C}, 62 \% ;{ }^{1} \mathrm{H}-\mathrm{NMR}\left(\mathrm{CDCl}_{3}, 400 \mathrm{MHz}\right): \delta(\mathrm{ppm})=0.61-0.65\left(\mathrm{~m}, 2 \mathrm{H}, \mathrm{CH}_{2}\right)$, 0.88-0.93 (m, 2H, CH 2$), 1.20\left(\mathrm{t}, 3 \mathrm{H}, \mathrm{CH}_{3}\right), 2.48-2.55\left(\mathrm{~m}, 2 \mathrm{H}, \mathrm{CH}_{2}\right), 2.70-2.74\left(\mathrm{~m}, 2 \mathrm{H}, \mathrm{CH}_{2}\right), 2.99-3.04$ (m, 1H, H-1), 3.14-3.20 (m, 1H, CH), 3.97-4.08 (m, 2H, OCH $), 5.30-5.38(\mathrm{~m}, 1 \mathrm{H}, \mathrm{H}-6), 5.91-5.98(\mathrm{~m}$, 2H, H-3, H-4), 6.04 (brs, 1H, N-H), 7.98 (s, 1H, Ar-H), 8.44 (s, 1H, Ar-H); ${ }^{13} \mathrm{C}-\mathrm{NMR}\left(\mathrm{CDCl}_{3}, 126 \mathrm{MHz}\right)$ : $\delta(\mathrm{ppm})=7.4,13.9,23.7,25.2,29.8,42.0,49.2,61.0,119.3,124.8,125.9,139.0,148.6,153.0,155.7,172.1$; MS (ES, pos) $m / z=328(\mathrm{M}+1)$.<smiles>CCOC(=O)C1CC=CCC1Nc1ncnc(Cl)c1N</smiles>

Ethyl (1S*,6S*)-6-((5-amino-6-chloropyrimidin-4-yl)amino)cyclohex-3-ene-1-carboxylate, $( \pm)$-29.

White solid, m.p. $120-121{ }^{\circ} \mathrm{C}, 42 \% ;{ }^{1} \mathrm{H}-\mathrm{NMR}\left(\mathrm{CDCl}_{3}, 400 \mathrm{MHz}\right): \delta(\mathrm{ppm})=1.19\left(\mathrm{t}, 3 \mathrm{H}, \mathrm{CH}_{3}, J=7.12 \mathrm{~Hz}\right)$, 2.03-2.12 (m, 1H, $\left.\mathrm{CH}_{2}\right), 2.34-2.43\left(\mathrm{~m}, 1 \mathrm{H}, \mathrm{CH}_{2}\right), 2.56-2.75\left(\mathrm{~m}, 2 \mathrm{H}, \mathrm{CH}_{2}\right), 2.83-2.92(\mathrm{~m}, 1 \mathrm{H}, \mathrm{H}-1), 3.47$ (brs, $\left.2 \mathrm{H}, \mathrm{NH}_{2}\right), 4.06-4.16\left(\mathrm{~m}, 2 \mathrm{H}, \mathrm{OCH}_{2}\right), 4.62-4.69(\mathrm{~m}, 1 \mathrm{H}, \mathrm{H}-6), 5.13(\mathrm{~d}, 1 \mathrm{H}, \mathrm{N}-\mathrm{H}, J=8.12 \mathrm{~Hz})$, 5.66-5.78 (m, 2H, H-3, H-4), 8.09 (s, 1H, Ar-H); ${ }^{13 \mathrm{C}}-\mathrm{NMR}\left(\mathrm{CDCl}_{3}, 100 \mathrm{MHz}\right): \delta(\mathrm{ppm})=14.5,27.5,31.9$, $45.2,48.5,61.3,122.1,124.2,125.1,143.7,150.1,154.8,174.1$; MS (ES, pos) $m / z=297(\mathrm{M}+1)$.<smiles>CCOC(=O)C1CC=CCC1n1cnc2c(Cl)ncnc21</smiles>

Ethyl $\left(1 S^{*}, 6 S^{*}\right)-6$-(6-chloro-9H-purin-9-yl)cyclohex-3-ene-1-carboxylate, $( \pm)$-30.

Colorless oil, 58\%; ${ }^{1} \mathrm{H}-\mathrm{NMR}\left(\mathrm{CDCl}_{3}, 400 \mathrm{MHz}\right): \delta(\mathrm{ppm})=0.96\left(\mathrm{t}, 3 \mathrm{H}, \mathrm{CH}_{3}, J=7.12 \mathrm{~Hz}\right), 2.53-2.68(\mathrm{~m}$, $\left.3 \mathrm{H}, \mathrm{CH}_{2}\right), 3.01-3.07\left(\mathrm{~m}, 1 \mathrm{H}, \mathrm{CH}_{2}\right), 3.56-3.66(\mathrm{~m}, 1 \mathrm{H}, \mathrm{H}-1), 3.86-3.96\left(\mathrm{~m}, 2 \mathrm{H}, \mathrm{OCH}_{2}\right), 4.94-5.03(\mathrm{~m}, 1 \mathrm{H}$, H-6), 5.78-5.89 (m, 2H, H-3, H-4), 8.17 (s, 1H, Ar-H), 8.76 (s, 1H, Ar-H); ${ }^{13} \mathrm{C}-\mathrm{NMR}\left(\mathrm{CDCl}_{3}, 100 \mathrm{MHz}\right)$ : $\delta(\mathrm{ppm})=14.2,29.0,30.7,44.1,54.5,61.4,124.4,125.6,132.2,145.3,151.5,151.9,152.1,173.0$; MS (ES, pos) $m / z=307(\mathrm{M}+1)$.<smiles>CCOC(=O)[C@H]1CC=CCC1n1cnc2c(N)ncnc21</smiles>

Ethyl $\left(1 S^{*}, 6 S^{*}\right)-6-(6-a z i d o-9 H$-purin-9-yl)cyclohex-3-ene-1-carboxylate, $( \pm)-31$.

White solid, m.p. $140-141{ }^{\circ} \mathrm{C}, 68 \%{ }^{1}{ }^{1} \mathrm{H}-\mathrm{NMR}$ (DMSO, $\left.400 \mathrm{MHz}\right): \delta(\mathrm{ppm})=0.74\left(\mathrm{t}, 3 \mathrm{H}, \mathrm{CH}_{3}, J=7.08 \mathrm{~Hz}\right.$ ), 2.46-2.57 (m, 3H, $\left.\mathrm{CH}_{2}\right), 2.86-3.00\left(\mathrm{~m}, 1 \mathrm{H}, \mathrm{CH}_{2}\right), 3.60-3.66(\mathrm{~m}, 1 \mathrm{H}, \mathrm{H}-1), 3.69-3.76\left(\mathrm{~m}, 2 \mathrm{H}, \mathrm{OCH}_{2}\right)$, 5.08-5.15 (m, 1H, H-6), 5.80-5.92 (m, 2H, H-3, H-4), 8.79 (s, Ar-H), 10.13 (s, 1H, Ar-H). ${ }^{13} \mathrm{C}-\mathrm{NMR}$ $(\mathrm{DMSO}, 100 \mathrm{MHz}): \delta(\mathrm{ppm})=14.3,29.3,31.9,44.7,54.3,61.1,125.1,125.9,136.5,139.3,142.9,144.6$, 146.3, 173.1. MS (ES, pos) $m / z=314(\mathrm{M}+1)$. 
<smiles>CCOC(=O)C1CCC=CC1Nc1ncnc(Cl)c1N</smiles>

Ethyl $\left(1 R^{*}, 2 S^{*}\right)$-2-((5-amino-6-chloropyrimidin-4-yl)amino)cyclohex-3-ene-1-carboxylate, $( \pm)$-34.

Brown oil, 55\%; ${ }^{1} \mathrm{H}-\mathrm{NMR}\left(\mathrm{CDCl}_{3}, 400 \mathrm{MHz}\right): \delta(\mathrm{ppm})=1.20\left(\mathrm{t}, 3 \mathrm{H}, \mathrm{CH}_{3}, J=7.14 \mathrm{~Hz}\right), 1.99-2.19(\mathrm{~m}$, $\left.4 \mathrm{H}, \mathrm{CH}_{2}\right), 2.99-3.05(\mathrm{~m}, 1 \mathrm{H}, \mathrm{H}-1), 4.00-4.17\left(\mathrm{~m}, 2 \mathrm{H}, \mathrm{OCH}_{2}\right), 5.23-5.26(\mathrm{~m}, 1 \mathrm{H}, \mathrm{H}-2), 5.59(\mathrm{~d}, 1 \mathrm{H}, \mathrm{N}-\mathrm{H}$,

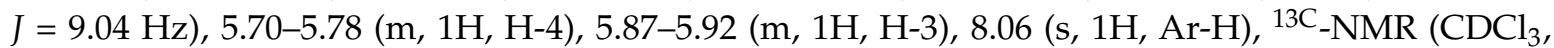
$100 \mathrm{MHz}): \delta(\mathrm{ppm})=14.5,22.7,23.6,43.4,46.9,61.1,122.4,127.5,130.0,143.4,149.7,154.6,174.2 ; \mathrm{MS}$ (ES, pos) $m / z=297(\mathbf{M}+1)$.<smiles>CCOC(=O)C1CCC=CC1n1cnc2c(Cl)ncnc21</smiles>

Ethyl $\left(1 R^{*}, 2 S^{*}\right)$-2-(6-chloro-9H-purin-9-yl)cyclohex-3-ene-1-carboxylate, $( \pm)$-35.

Brown solid, m.p. $119-121^{\circ} \mathrm{C}, 68 \% ;{ }^{1} \mathrm{H}-\mathrm{NMR}\left(\mathrm{CDCl}_{3}, 400 \mathrm{MHz}\right): \delta(\mathrm{ppm})=1.03\left(\mathrm{t}, 3 \mathrm{H}, \mathrm{CH}_{3}, J=7.14 \mathrm{~Hz}\right)$, 2.00-2.08 (m, 2H, $\left.\mathrm{CH}_{2}\right), 2.27-2.33\left(\mathrm{~m}, 1 \mathrm{H}, \mathrm{CH}_{2}\right), 2.41-2.46\left(\mathrm{~m}, 1 \mathrm{H}, \mathrm{CH}_{2}\right), 3.12-3.20(\mathrm{~m}, 1 \mathrm{H}, \mathrm{H}-1)$, 3.72-3.87 (m, 2H, OCH $)_{2}, 5.67-5.70(\mathrm{~m}, 1 \mathrm{H}, \mathrm{H}-2), 5.83-5.90(\mathrm{~m}, 1 \mathrm{H}, \mathrm{H}-4), 6.28-6.33(\mathrm{~m}, 1 \mathrm{H}, \mathrm{H}-3), 8.23$ (s, 1H, Ar-H), 8.73 (s, 1H, Ar-H); ${ }^{13} \mathrm{C}-\mathrm{NMR}(\mathrm{DMSO}, 100 \mathrm{MHz}): \delta(\mathrm{ppm})=14.3,20.3,24.4,44.1,50.1$, $61.0,123.1,134.8,147.5,147.8,149.9,152.3,152.9,172.4$; MS (ES, pos) $m / z=307(\mathrm{M}+1), 309(\mathrm{M}+3)$.<smiles>CCOC(=O)C1CC=CC1Nc1ncnc(Cl)c1N</smiles>

Ethyl (1 $\left.R^{*}, 2 S^{*}\right)$-2-((5-amino-6-chloropyrimidin-4-yl)amino)cyclopent-3-ene-1-carboxylate, $( \pm)$-38.

Brownish white solid, m.p. $104-106{ }^{\circ} \mathrm{C}, 34 \% ;{ }^{1} \mathrm{H}-\mathrm{NMR}\left(\mathrm{CDCl}_{3}, 400 \mathrm{MHz}\right): \delta(\mathrm{ppm})=1.04\left(\mathrm{t}, 3 \mathrm{H}, \mathrm{CH}_{3}\right.$, $J=7.12 \mathrm{~Hz}), 2.56-2.67\left(\mathrm{~m}, 1 \mathrm{H}, \mathrm{CH}_{2}\right), 2.83-2.90\left(\mathrm{~m}, 1 \mathrm{H}, \mathrm{CH}_{2}\right), 3.44-3.54(\mathrm{~m}, 1 \mathrm{H}, \mathrm{H}-1), 3.57$ (brs, $2 \mathrm{H}$, $\left.\mathrm{NH}_{2}\right), 3.85-4.02\left(\mathrm{~m}, 2 \mathrm{H}, \mathrm{OCH}_{2}\right), 5.34(\mathrm{~d}, 1 \mathrm{H}, \mathrm{N}-\mathrm{H}, J=8.76 \mathrm{~Hz}), 5.69-5.76(\mathrm{~m}, 2 \mathrm{H}, \mathrm{H}-2, \mathrm{H}-4), 5.98-6.01$ $(\mathrm{m}, 1 \mathrm{H}, \mathrm{H}-3), 8.06$ (s, 1H, Ar-H); ${ }^{13} \mathrm{C}-\mathrm{NMR}\left(\mathrm{CDCl}_{3}, 100 \mathrm{MHz}\right): \delta(\mathrm{ppm})=14.2,35.4,46.4,58.2,61.1$, 122.6, 130.2, 134.0, 143.0, 149.3, 154.1, 174.0; MS (ES, pos) $m / z=283(\mathrm{M}+1), 285(\mathrm{M}+3)$.<smiles>CCOC(=O)C1CC=CC1n1cnc2c(Cl)ncnc21</smiles>

Ethyl (1R*,2S*)-2-(6-chloro-9H-purin-9-yl)cyclopent-3-ene-1-carboxylate, $( \pm)$-39.

Yellowish white solid, m.p. $118-119{ }^{\circ} \mathrm{C}, 39 \% ;{ }^{1} \mathrm{H}-\mathrm{NMR}\left(\mathrm{CDCl}_{3}, 400 \mathrm{MHz}\right): \delta(\mathrm{ppm})=0.72\left(\mathrm{t}, 3 \mathrm{H}, \mathrm{CH}_{3}\right.$, $J=7.14 \mathrm{~Hz}), 2.76-2.85\left(\mathrm{~m}, 1 \mathrm{H}, \mathrm{CH}_{2}\right), 3.14-3.23\left(\mathrm{~m}, 1 \mathrm{H}, \mathrm{CH}_{2}\right), 3.49-3.56(\mathrm{~m}, 1 \mathrm{H}, \mathrm{H}-1), 3.65-3.77(\mathrm{~m}, 2 \mathrm{H}$, $\left.\mathrm{OCH}_{2}\right), 5.86-5.89(\mathrm{~m}, 1 \mathrm{H}, \mathrm{H}-2), 5.15-5.17$ (m, 1H, H-4), 6.44-6.47 (m, 1H, H-3), 7.99-8.01 (m, 1H, Ar-H), 8.78-8.81 (m, $1 \mathrm{H}, \mathrm{Ar}-\mathrm{H}) ;{ }^{13} \mathrm{C}-\mathrm{NMR}\left(\mathrm{CDCl}_{3}, 100 \mathrm{MHz}\right): \delta(\mathrm{ppm})=13.8,34.9,47.2,60.9,61.4,126.8,134.7$, 138.7, 150.0, 152.2, 152.3, 154.2, 170.9; MS (ES, pos) $m / z=293(\mathrm{M}+1), 295(\mathrm{M}+3)$. 
<smiles>Nc1c(Cl)ncnc1NC1CC=CCC1CO</smiles>

$\left(\left(1 R^{*}, 6 S^{*}\right)-6-((5-A m i n o-6-c h l o r o p y r i m i d i n-4-y l) a m i n o) c y c l o h e x-3-e n-1-y l\right) m e t h a n o l, ~( \pm)-42$.

White solid, m.p. $186-188^{\circ} \mathrm{C}, 60 \%{ }^{1}{ }^{1} \mathrm{H}-\mathrm{NMR}$ (DMSO, $\left.400 \mathrm{MHz}\right): \delta(\mathrm{ppm})=1.96-2.12\left(\mathrm{~m}, 4 \mathrm{H}, \mathrm{CH}_{2}\right)$, 2.21-2.30 (m, 1H, H-1), 3.24-3.32 (m, 1H, OCH $), 3.40-3.49\left(\mathrm{~m}, 1 \mathrm{H}, \mathrm{OCH}_{2}\right), 4.43-4.48(\mathrm{~m}, 2 \mathrm{H}, \mathrm{H}-6$ and O-H), 5.16 (brs, 2H, N-H), 5.59-5.70 (m, 2H, H-3, H-4), 6.24 (d, 1H, N-H, J = 7.56 Hz), 7.68 (s, 1H, Ar-H); ${ }^{13} \mathrm{C}-\mathrm{NMR}(\mathrm{DMSO}, 100 \mathrm{MHz}): \delta(\mathrm{ppm})=25.9,30.1,39.8,47.2,61.4,124.5,125.6,126.6,138.0,146.4,152.6$; MS (ES, pos) $m / z=255(\mathrm{M}+1), 257(\mathrm{M}+3)$.<smiles>OCC1CC=CCC1n1cnc2c(Cl)ncnc21</smiles>

$\left(\left(1 R^{*}, 6 S^{*}\right)-6-(6-C h l o r o-9 H-p u r i n-9-y l) c y c l o h e x-3-e n-1-y l\right) m e t h a n o l, ~( \pm)-43$.

White solid, m.p. ${ }^{109-111}{ }^{\circ} \mathrm{C}, 76 \%,{ }^{1} \mathrm{H}-\mathrm{NMR}\left(\mathrm{CDCl}_{3}, 500 \mathrm{MHz}\right): \delta(\mathrm{ppm})=1.36-1.47\left(\mathrm{~m}, 1 \mathrm{H}, \mathrm{CH}_{2}\right)$, 2.05-2.15 (m, 1H, CH $\mathrm{CH}_{2}, 2.34-2.44(\mathrm{~m}, 1 \mathrm{H}, \mathrm{H}-1), 2.50-2.59\left(\mathrm{~m}, 1 \mathrm{H}, \mathrm{CH}_{2}\right), 2.73-2.81\left(\mathrm{~m}, 1 \mathrm{H}, \mathrm{OCH}_{2}\right)$, 2.93-3.03 (m, 1H, CH $\left.\mathrm{CH}_{2}\right), 3.44-3.53\left(\mathrm{~m}, 1 \mathrm{H}, \mathrm{OCH}_{2}\right), 4.65(\mathrm{brs}, 1 \mathrm{H}, \mathrm{OH}), 5.31-5.37(\mathrm{~m}, 1 \mathrm{H}, \mathrm{H}-6), 5.98-6.06$ (m, 2H, H-3, H-4), 8.33 (s, 1H, Ar-H), 8.76 (s, 1H, Ar-H); ${ }^{13} \mathrm{C}-\mathrm{NMR}\left(\mathrm{CDCl}_{3}, 126 \mathrm{MHz}\right): 22.6,31.0,39.7$, $48.6,62.1,124.7,127.8,131.1,144.6,151.6,151.7,152.5$, MS (ES, pos) $m / z=265(\mathrm{M}+1), 267(\mathrm{M}+3)$.<smiles>Nc1c(Cl)ncnc1NC1CC=CC[C@H]1CO</smiles>

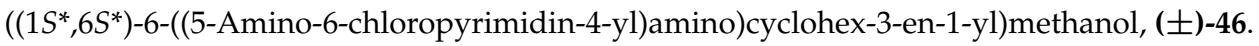

White solid, m.p. $164-167{ }^{\circ} \mathrm{C}, 64 \%{ }^{1} \mathrm{H}-\mathrm{NMR}(\mathrm{DMSO}, 400 \mathrm{MHz}): \delta(\mathrm{ppm})=1.70-1.84\left(\mathrm{~m}, 1 \mathrm{H}, \mathrm{CH}_{2}\right)$, 1.89-2.05 (m, 2H, CH $\left.\mathrm{CH}_{2}\right), 2.14-2.25\left(\mathrm{~m}, 1 \mathrm{H}, \mathrm{CH}_{2}\right), 2.26-2.36(\mathrm{~m}, 1 \mathrm{H}, \mathrm{H}-1), 3.26-3.43\left(\mathrm{~m}, 2 \mathrm{H}, \mathrm{OCH}_{2}\right)$, 4.05-4.15 (m, 1H, H-6), $4.36(\mathrm{t}, 1 \mathrm{H}, \mathrm{O}-\mathrm{H}, J=5.32 \mathrm{~Hz}), 4.99$ (brs, 2H, N-H), 5.51-5.67 (m, 2H, H-3, H-4), $6.50(\mathrm{~d}, 1 \mathrm{H}, \mathrm{N}-\mathrm{H}, J=7.96 \mathrm{~Hz}), 7.64(\mathrm{~s}, 1 \mathrm{H}, \mathrm{Ar}-\mathrm{H}),{ }^{13} \mathrm{C}-\mathrm{NMR}(\mathrm{DMSO}, 100 \mathrm{MHz}): \delta(\mathrm{ppm})=28.7,32.3$, $41.3,48.3,62.9,124.2,125.5,127.3,137.7,146.4,152.7$, MS (ES, pos) $m / z=255(\mathrm{M}+1), 257(\mathrm{M}+3)$.<smiles>OC[C@H]1CC=CC[C@@H]1n1cnc2c(Cl)ncnc21</smiles>

$\left(\left(1 S^{*}, 6 S^{*}\right)-6-(6-C h l o r o-9 H-p u r i n-9-y l) c y c l o h e x-3-e n-1-y l\right) m e t h a n o l, ~( \pm)-47$.

White solid, m.p. $160-162{ }^{\circ} \mathrm{C}, 70 \% ;{ }^{1} \mathrm{H}-\mathrm{NMR}$ (DMSO, $\left.500 \mathrm{MHz}\right): \delta(\mathrm{ppm})=2.13-2.29\left(\mathrm{~m}, 2 \mathrm{H}, \mathrm{CH}_{2}\right)$, 2.42-2.49 (m, 1H, $\left.\mathrm{CH}_{2}\right), 2.53-2.62(\mathrm{~m}, 1 \mathrm{H}, \mathrm{H}-1), 2.83-2.95\left(\mathrm{~m}, 1 \mathrm{H}, \mathrm{CH}_{2}\right), 2.98-3.05\left(\mathrm{~m}, 1 \mathrm{H}, \mathrm{OCH}_{2}\right)$, 3.11-3.17 (m, 1H, $\left.\mathrm{OCH}_{2}\right), 4.69-4.78(\mathrm{~m}, 1 \mathrm{H}, \mathrm{H}-6), 5.68-5.85(\mathrm{~m}, 2 \mathrm{H}, \mathrm{H}-3, \mathrm{H}-4), 8.73-8.78(\mathrm{~m}, 2 \mathrm{H}, \mathrm{Ar}-\mathrm{H})$, ${ }^{13} \mathrm{C}-\mathrm{NMR}$ (DMSO, $\left.126 \mathrm{MHz}\right): 28.5,31.3,39.0,54.1,61.7,124.6,127.2,131.6,147.6,149.4,151.7,152.4 ; \mathrm{MS}$ (ES, pos) $m / z=265(\mathrm{M}+1), 267(\mathrm{M}+3)$. 
Supplementary Materials: The Supplementary Materials are available online.

Author Contributions: Both authors equally contributed to the manuscript. L.K. designed the experiments. A.R. performed the analyses and wrote the paper. L.K. reviesed the manuscript.

Funding: We are grateful to the Hungarian Research Foundation (NKFIH No. K 119282) for financial support. The financial support of the GINOP-2.3.2-15-2016-00034 project is also acknowledged. This research was supported by the EU-funded Hungarian grant EFOP-3.6.1-16-2016-00008. Ministry of Human Capacities, Hungary grant 20391-3/2018/FEKUSTRAT is also acknowledged.

Conflicts of Interest: The authors declare no conflict of interest.

\section{References}

1. Rodríguez, J.B.; Comin, M.J. New progresses in the enantioselective synthesis and biological properties of carbocyclic nucleosides. Mini-Rev. Med. Chem. 2003, 3, 95-114. [CrossRef] [PubMed]

2. Weising, S.; Dekiert, P.; Schols, D.; Neyts, J.; Meier, C. Synthesis of enantiomerically pure $1^{\prime}, 2^{\prime}$-cis-dideoxy, -dideoxydi-dehydro, -ribo and -deoxy carbocyclic nucleoside analogues. Synthesis 2018, 50, 2266-2280.

3. Casu, F.; Chiacchio, M.A.; Romeo, R.; Gumina, G. Chiral synthesis of carbocyclic nucleoside analogs from noncarbohydrate precursors. Curr. Org. Chem. 2007, 11, 999-1016. [CrossRef]

4. Wang, J.; Rawal, R.K.; Chu, C.K. Recent advances in carbocyclic nucleosides: Synthesis and biological activity. In Medicinal Chemistry of Nucleic Acids; Zhang, L.H., Xi, Z., Chattopadhyaya, J., Eds.; John Wiley: New York, NY, USA, 2011; pp. 1-100.

5. Boutureira, O.; Matheu, M.I.; Díaz, Y.; Castillón, S. Advances in the enantioselective synthesis of carbocyclic nucleosides. Chem. Soc. Rev. 2013, 42, 5056-5072. [CrossRef] [PubMed]

6. Seley-Radtke, K.L.; Yates, M.K. The evolution of nucleoside analogue antivirals: A review for chemists and non-chemists. Part 1: Early structural modifications to the nucleoside scaffold. Antivir. Res. 2018, 154, 66-86. [CrossRef] [PubMed]

7. Wang, J.; Froeyen, M.; Hendrix, C.; Andrei, G.; Snoeck, R.; De Clercq, E.; Herdewijn, P. The cyclohexene ring system as a furanose mimic: Synthesis and antiviral activity of both enantiomers of cyclohexenylguanine. J. Med. Chem. 2000, 43, 736-745. [CrossRef] [PubMed]

8. Barral, K.; Courcambeck, J.; Pèpe, G.; Balzarini, J.; Neyts, J.; De Clercq, E.; Camplo, M. Synthesis and antiviral evaluation of cis-substituted cyclohexenyl and cyclohexanyl nucleosides. J. Med. Chem. 2005, 48, 450-456. [CrossRef] [PubMed]

9. Viña, D.; Santana, L.; Uriarte, E.; Terán, C. 1,2-Disubstituted cyclohexane nucleosides: Comparative study for the synthesis of cis and trans adenosine analogues. Tetrahedron 2005, 61, 473-478. [CrossRef]

10. Kiss, L.; Fülöp, F. Synthesis of carbocyclic and heterocyclic $\beta$-aminocarboxylic acids. Chem. Rev. 2014, 114, 1116-1169. [CrossRef]

11. Kiss, L.; Mándity, I.M.; · Fülöp, F. Highly functionalized cyclic $\beta$-amino acid moieties as promising scaffolds in peptide research and drug design. Amino Acids 2017, 49, 1441-1455. [CrossRef]

12. Juaristi, E.; Soloshonok, V. Enantioselective Synthesis of $\beta$-Amino Acids, 2nd ed.; Wiley: Hoboken, NJ, USA, 2005.

13. Risseeuw, M.; Overhand, M.; Fleet, G.W.J.; Simone, M.I. A compendium of cyclic sugar amino acids and their carbocyclic and heterocyclic nitrogen analogues. Amino Acids 2013, 45, 613-689. [CrossRef]

14. Fülöp, F.; Martinek, T.A.; Tóth, G.K. Application of alicyclic $\beta$-amino acids in peptide chemistry. Chem. Soc. Rev. 2006, 35, 323-334. [CrossRef]

15. Timoshchuk, V.A. Nucleosides of uronic acids as a component of natural antibiotics. Pharm. Chem. J. 1995, 29, 281-289. [CrossRef]

16. Chandrasekhar, S.; Kiranmai, N.; Kiran, M.U.; Devi, A.S.; Reddy, G.P.K.; Idris, M.; Jagadeesh, B. Novel helical foldamers: Organized heterogeneous backbone folding in 1:1 $\alpha /$ nucleoside-derived- $\beta$-amino acid sequences. Chem. Commun. 2010, 46, 6962-6964. [CrossRef] [PubMed]

17. Hausler, N.E.; Devine, S.M.; McRobb, F.M.; Warfe, L.; Pouton, C.W.; Haynes, J.M.; Bottle, S.E.; White, P.J.; Scammells, P.J. Synthesis and Pharmacological Evaluation of Dual Acting Antioxidant $\mathrm{A}_{2 \mathrm{~A}}$ Adenosine Receptor Agonists. J. Med. Chem. 2012, 55, 3521-3534. [CrossRef] [PubMed]

18. Porter, E.A.; Weisblum, B.; Gellman, S.H. Mimicry of host-defense peptides by unnatural oligomers: Antimicrobial $\beta$-peptides. J. Am. Chem. Soc. 2002, 124, 7324-7330. [CrossRef] [PubMed] 
19. Haase, H.S.; Peterson-Kaufman, K.J.; Levengood, S.K.L.; Checco, J.W.; Murphy, W.L.; Gellman, S.H. Extending Foldamer Design beyond $\alpha$-Helix Mimicry: $\alpha / \beta$-Peptide Inhibitors of Vascular Endothelial Growth Factor Signaling. J. Am. Chem. Soc. 2012, 134, 7652-7655. [CrossRef]

20. Mayans, E.; Gargallo, A.; Álvarez-Larena, A.; Illa, O.; Ortuño, R.M. Diastereodivergent Synthesis of Chiral vic-Disubstituted-Cyclobutane Scaffolds: 1,3-Amino Alcohol and 1,3-Diamine Derivatives-Preliminary Use in Organocatalysis. Eur. J. Org. Chem. 2013, 2013, 1425-1433. [CrossRef]

21. Choi, S.H.; Ivancic, M.; Guzei, I.A.; Gellman, S.H. Structural Characterization of Peptide Oligomers Containing (1R,2S)-2-Aminocyclohexanecarboxylic Acid (cis-ACHC). Eur. J. Org. Chem. 2013, 2013, 3464-3469. [CrossRef]

22. Laborda, P.; Wang, S.-Y.; Voglmeir, J. Influenza neuraminidase inhibitors: Synthetic approaches, derivatives and biological activity. Molecules 2016, 21, 1513. [CrossRef]

23. Goa, K.L.; Sorkin, E.M. Gabapentin. A review of its pharmacological properties and clinical potential in epilepsy. Drugs 1993, 46, 409-427. [CrossRef] [PubMed]

24. Silverman, R.B. The 2011 E. B. Hershberg Award for important discoveries in medicinally active substances: (1S,3S)-3-amino-4-difluoromethylenyl-1-cyclopentanoic acid (CPP-115), a GABA aminotransferase inactivator and new treatment for drug addiction and infantile spasms. J. Med. Chem. 2012, 55, 567-575. [CrossRef] [PubMed]

25. Vince, R.; Hua, M.; Brownell, J.; Daluge, S.; Lee, F.; Shannon, W.M.; Lavelle, G.C.; Qualls, J.; Weislow, O.S.; Kiser, R.; et al. Potent and selective activity of a new carbocyclic nucleoside analog (carbovir: NSC 614846) against human immunodeficiency virus in vitro. Biochem. Biophys. Res. Commun. 1988, 156, 1046-1053. [CrossRef]

26. Karlsson, S.; Cornwall, P.; Cruz, A.; Pontén, F.; Fridén-Saxin, M.; Turner, A. Diastereoselective 1,4-Conjugate Addition of Alkyl Cuprates to Methyl Cyclopent-1-enecarboxylates. Org. Process Res. Dev. 2018, 22, 337-343. [CrossRef]

27. Singh, R.; Vince, R. 2-Azabicyclo[2.2.1]hept-5-en-3-one: Chemical profile of a versatile synthetic building block and its impact on the development of therapeutics. Chem. Rev. 2012, 112, 4642-4686. [CrossRef]

28. Kiss, L.; Forró, E.; Sillanpää, R.; Fülöp, F. Synthesis of conformationally restricted 1,2,3-triazole-substituted ethyl $\beta$ - and $\gamma$-aminocyclopentanecarboxylate stereoisomers. Multifunctionalized alicyclic amino esters. Tetrahedron 2010, 66, 3599-3607. [CrossRef]

29. Kiss, L.; Forró, E.; Martinek, T.A.; Bernáth, G.; De Kimpe, N.; Fülöp, F. Stereoselective synthesis of hydroxylated $\beta$-aminocyclohexanecarboxylic acids. Tetrahedron 2008, 64, 5036-5043. [CrossRef]

30. Kiss, L.; Forró, E.; Orsy, G.; Ábrahámi, R.A.; Fülöp, F. Stereo- and regiocontrolled syntheses of exomethylenic cyclohexane $\beta$-amino acid derivatives. Molecules 2015, 20, 21094-21102. [CrossRef]

31. Kiss, L.; Forró, E.; Fülöp, F. A new strategy for the regio- and stereoselective hydroxylation of trans-2-aminocyclohexenecarboxylic acid. Tetrahedron Lett. 2006, 47, 2855-2858. [CrossRef]

32. Bernáth, G.; Stájer, G.; Szabó, A.E.; Fülöp, F. Stereochemical studies 83 saturated heterocycles 76: Preparation and conformational study of partially saturated 3,1-benzoxazines, 3,1-benzoxazin-2-ones and 3,1-benzoxazine-2-thiones. Tetrahedron 1985, 41, 1353-1365. [CrossRef]

33. Kazi, B.; Kiss, L.; Forró, E.; Fülöp, F. Synthesis of orthogonally protected azepane $\beta$-amino ester enantiomers. Tetrahedron Lett. 2010, 51, 82-85. [CrossRef]

34. Kiss, L.; Forró, E.; Fustero, S.; Fülöp, F. Regio- and diastereoselective fluorination of alicyclic $\beta$-amino acids. Org. Biomol. Chem. 2011, 9, 6528-6534. [CrossRef] [PubMed]

35. Kiss, L.; Kardos, M.; Vass, C.; Fülöp, F. Application of metathesis reactions in the synthesis and transformations of functionalized $\beta$-amino acid derivatives. Synthesis 2018, 50, 3571-3588. [CrossRef]

36. Kiss, L.; Fülöp, F. Selective synthesis of fluorine-containing cyclic $\beta$-amino acid scaffolds. Chem. Rec. 2018, 18, 266-281. [CrossRef]

37. Nonn, M.; Remete, A.M.; Fülöp, F.; Kiss, L. Recent advances in the transformations of cycloalkane-fused oxiranes and aziridines. Tetrahedron 2017, 73, 5461-5483. [CrossRef]

38. Ábrahámi, R.A.; Kiss, L.; Fustero, S.; Fülöp, F. Functionalized dialdehydes as promising scaffolds for access to heterocycles and $\beta$-amino acids: Synthesis of fluorinated piperidine and azepane derivatives. Synthesis 2017, 49, 1206-1213. 
39. Forró, E.; Fülöp, F. Enzymatic Method for the Synthesis of Blockbuster Drug Intermediates -Synthesis of Five-Membered Cyclic $\gamma$-Amino Acid and $\gamma$-Lactam Enantiomers. Eur. J. Org. Chem. 2008, 45, 5263-5268. [CrossRef]

40. Forró, E.; Kiss, L.; Árva, J.; Fülöp, F. Efficient Enzymatic Routes for the Synthesis of New Eight-membered Cyclic beta-Amino Acid and beta-Lactam Enantiomers. Molecules 2018, 22, 2221. [CrossRef]

41. Forró, E.; Megyesi, R.; Paál, T.A.; Fülöp, F. Efficient dynamic kinetic resolution method for the synthesis of enantiopure 6-hydroxy- and 6-methoxy-1,2,3,4-tetrahydroisoquinoline-1-carboxylic acid. Tetrahedron Asymmetry 2016, 27, 1213-1216. [CrossRef]

42. Kiss, L.; Forró, E.; Sillanpää, R.; Fülöp, F. Diastereo- and enantioselective synthesis of orthogonally protected 2,4-diaminocyclopentanecarboxylates: A flip from $\beta$-amino- to $\beta, \gamma$-diaminocarboxylates. J. Org. Chem. 2007, 72, 8786-8790. [CrossRef]

Sample Availability: Samples of all compounds are available in mg amounts from the authors.

(C) 2019 by the authors. Licensee MDPI, Basel, Switzerland. This article is an open access article distributed under the terms and conditions of the Creative Commons Attribution (CC BY) license (http:/ / creativecommons.org/licenses/by/4.0/). 